\title{
Creating nuclear spin entanglement using an optical degree of freedom
}

\author{
Marcus Schaffry, ${ }^{1}$ Brendon W. Lovett, ${ }^{1,2}$ and Erik M. Gauger ${ }^{1,3, *}$ \\ ${ }^{1}$ Department of Materials, Oxford University, Oxford OX1 3PH, United Kingdom \\ ${ }^{2}$ SUPA, Department of Physics, Heriot Watt University, Edinburgh EH14 4AS, United Kingdom \\ ${ }^{3}$ Centre for Quantum Technologies, National University of Singapore, 3 Science Drive 2, Singapore 117543
}

(Received 24 June 2011; published 21 September 2011)

\begin{abstract}
Molecular nanostructures are promising building blocks for future quantum technologies, provided methods of harnessing their multiple degrees of freedom can be identified and implemented. Due to low decoherence rates, nuclear spins are considered ideal candidates for storing quantum information, while optical excitations can give rise to fast and controllable interactions for information processing. A recent paper [M. Schaffry et al., Phys. Rev. Lett. 104, 200501 (2010)] proposed a method for entangling two nuclear spins through their mutual coupling to a transient optically excited electron spin. Building on the same idea, we present here an extended and much more detailed theoretical framework, showing that this method is in fact applicable to a much wider class of molecular structures than previously discussed in the original proposal.
\end{abstract}

DOI: 10.1103/PhysRevA.84.032332

PACS number(s): 03.67.Bg, 03.67.Lx

\section{INTRODUCTION}

The controlled generation of entanglement is a crucial task in quantum computing, quantum cryptography, quantum error correction, and other quantum technologies. Experimentally, controlled entangling operations have been demonstrated in a wide range of systems including, among many others, pairs of photons [1], pairs of atoms [2], pairs of ions [3], between an atom and a photon [4], and between an ion and a photon [5]. However, a different approach typically needs to be used for each of these systems, so that a given control method may not readily be transferred to a different physical system.

Employing molecular systems as "quantum hardware" [6,7] offers the advantages of high reproducibility, chemically engineered system properties, and the potential for selfassembly into more complex functional units. In general, nuclear spins are widely considered to be the most promising quantum bits (qubits) for a quantum memory in solid-state systems, since they have remarkably long coherence times [8]. However, they are also hard to manipulate on a fast time scale, as nuclei are usually only weakly coupled. Hence the key challenge for nuclear spin-spin entanglement is achieving fast and switchable control over the interactions between adjacent qubits.

Several previous publications have proposed the introduction of a mediator spin, whose mutual coupling to the qubit spins provides a route for a controlled entangling operation [9-13]. In these studies the mediator spin is usually of the same type as the qubit spins and possesses a spin of $1 / 2$, so the system comprises either three electron or three nuclear spins. However, employing a mediator spin of the same kind as the qubit spins often does not allow for operation times that are many orders of magnitude shorter than the coherence time of the qubits. By contrast, many molecular structures possess transient optically excited triplet states (i.e., states with spin-1 character) that could be used as mediators for nearby nuclear spins [14-18]. In this article we show how fast controlled entangling operations with a high fidelity are possible in

\footnotetext{
*erik.gauger@materials.ox.ac.uk
}

suitable structures of this type. This constitutes an instance of a so-called hybrid approach to quantum computing [19], where the advantages of different qubit systems are combined to improve overall performance. In fact, the systems we consider here possess three distinct degrees of freedom: an optical one, an electron spin, and a nuclear spin. We believe that a future quantum processor will also likely harness a similar hierarchy of different degrees of freedom, providing additional motivation to probe the interplay between different degrees of freedom beyond the immediate purpose of this work.

A hybrid approach such as the one considered here offers several further advantages besides achieving fast gating times. For example, the presence of the optical excitation in this class of system may facilitate dynamic nuclear polarization $[20,21]$. Further, recent experiments have shown that the principal decoherence mechanism for nuclear spins that are controlled through their interaction with electron spins is caused by that very same interaction [22]. However, for the entangling operations that are the subject of the present article, the electron spin is transient and only exists as long as the system is in its optically excited state, which should therefore be beneficial for the nuclear spin coherence time. Finally, the fact that the interaction is controllable implies that we do not rely on dynamic decoupling schemes to switch off the interactions.

This paper is organised as follows: in Sec. II we introduce the most general Hamiltonian for our system and discuss several possible physical implementations. Section III analyzes the Hamiltonian for certain symmetry assumptions and for the general case. The symmetry considerations lead us to simplified effective Hamiltonians, whose dynamical properties are more easily accessible. We proceed by presenting appropriate protocols for the controlled generation of entanglement for different parameter regimes in Sec. IV. We also benchmark the performance of these protocols with respect to the predominant decoherence mechanisms. Finally, Sec. V contains a summary and discussion of our results. Throughout this paper, we closely relate our general ideas to the recent experiments $[23,24]$ which have motivated our analysis, but we would like to point out that our results are more widely applicable and not limited to these specific systems. 


\section{MODEL}

For our model we consider a structure comprising three integral components: two nuclear spin qubits, labeled $n$ and $n^{\prime}$, and a mediator spin system with an optical degree of freedom. We denote the ground state of the mediator $|0\rangle$ and the first excited state $|e\rangle$. Further, $|0\rangle$ is assumed to be spin-silent, while $|e\rangle$ possesses an electronic spin-1 character. The spin-1 (quasi)particle is thus created upon optical excitation of the mediator system. Importantly, the two spin qubits do not directly interact with each other, yet both are coupled to the excited state of the mediator with an isotropic Heisenberg interaction as depicted schematically in Fig. 1. The Hamiltonian for such a three-particle system in an external magnetic field is then given by

$$
\begin{aligned}
H= & -\omega_{n} S_{z, n}-\omega_{n^{\prime}} S_{z, n^{\prime}}+|e\rangle\left(\omega_{e} S_{z, e}+\omega_{0}\right)\langle e| \\
& +|e\rangle\left(A \mathbf{S}_{n} \cdot \mathbf{S}_{e}+A^{\prime} \mathbf{S}_{n^{\prime}} \cdot \mathbf{S}_{e}+D S_{z, e}^{2}\right)\langle e|,
\end{aligned}
$$

where $\omega_{n / n^{\prime}}$ denotes the Zeeman splitting of the two qubits and $\omega_{e}$ that of the central spin-1 particle; $D$ is the uniaxial zerofield splitting, $A$ and $A^{\prime}$ are the isotropic Heisenberg coupling constants, and $\omega_{0}$ denotes the (typically optical) excitation energy of the mediator. Here $S_{z, n / n^{\prime} / e}$ and $\mathbf{S}_{n / n^{\prime} / e}$ are the usual component and total vector Pauli spin operators, respectively.

Hamiltonian (1) can describe many different nanostructures, in particular, a range of optically active molecules, in which case the mediator ground (excited) state corresponds to the absence (presence) of an electron-hole pair. The transition between these two states can be induced by a short laser pulse of frequency $\omega_{0}$; moreover, the excited state will decay naturally through spontaneous emission. By contrast, a nitrogen-vacancy center in diamond surrounded by two ${ }^{13} \mathrm{C}$ atoms [25] is an example of a system in which the spin-1 mediator is not susceptible to decay and is ever present. Many of the results discussed in this article are equally valid for this kind of system. However, the following discussion primarily focuses on the example case of the molecular system consisting of a functionalized $\mathrm{C}_{60}$ molecule with two functional groups as depicted in Fig. 2.

In what follows we first analyze the Hamiltonian, Eq. (1), for two cases: that of a symmetric and that of an asymmetric system. In order to understand the system properties and dynamics, we determine the eigensystem using perturbation theory. This will provide us with the necessary basis to design suitable protocols for the controlled generation of nuclear spin entanglement for both these cases. (a)

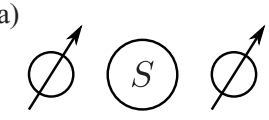

ground state (b)

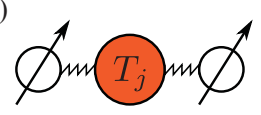

excited state

FIG. 1. (Color online) (a) There is no coupling between the mediator and the two nuclear spins in the ground (or vacuum) state $|0\rangle$. (b) The excited state of the mediator (e.g., an electron-hole pair) has spin-1 character and couples to both nuclear spins.

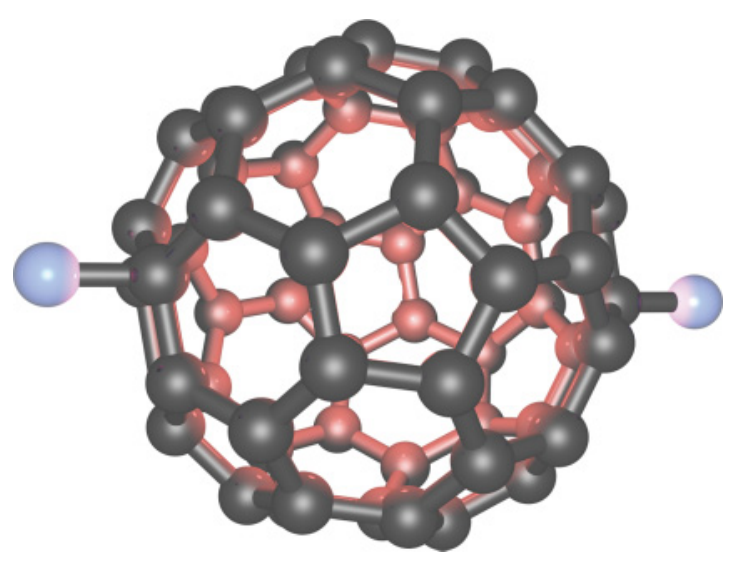

FIG. 2. (Color online) A functionalized buckyball with two satellite atoms [far left and far right (blue) spheres]. In reality the functional groups are likely much larger than a single atom, but for simplicity we depict here only the two relevant atoms with a nuclear spin $1 / 2$, for example, ${ }^{13} \mathrm{C},{ }^{15} \mathrm{~N}$, or ${ }^{31} \mathrm{P}$. Note that the two satellites (or, rather, functional groups) are not necessarily both the same and that the system may thus be "asymmetric."

\section{EIGENSPECTRUM AND EFFECTIVE HAMILTONIAN}

\section{A. Symmetric system}

By definition, the symmetric system consists of two nuclear spins with $\omega_{n}=\omega_{n^{\prime}}$ and equal hyperfine coupling constants $A=A^{\prime}$. In the presence of the electronic excitation, i.e., after the application of a suitable laser pulse, the Hamiltonian governing the spin dynamics is given by

$$
\begin{aligned}
H_{\mathrm{sym}}= & \langle e|H| e\rangle=-\omega_{n} S_{z, n}+\omega_{e} S_{z, e}-\omega_{n} S_{z, n^{\prime}} \\
& +A\left(\mathbf{S}_{n} \cdot \mathbf{S}_{e}+\mathbf{S}_{n^{\prime}} \cdot \mathbf{S}_{e}\right)+D S_{z, e}^{2},
\end{aligned}
$$

where we have neglected the term $\omega_{0}$, which is proportional to the identity and unimportant for the dynamics. Since the electronic Zeeman splitting $\omega_{e}$ is typically the highest energy scale of the system (and, in particular, much higher than $\omega_{n}$ ), it is safe to assume that

$$
\left|\omega_{n}\right|,|D|, A \ll \omega_{e} .
$$

Based on the above assumption, we employ degenerate perturbation theory to determine the eigenspectrum of $H_{\text {sym }}$. We begin by partitioning the Hamiltonian as

$$
H_{\text {sym }}=H_{0, \text { sym }}+H_{\text {sym }}^{\prime},
$$

where

$$
H_{0, \mathrm{sym}}=-\omega_{n} S_{z, n}+\omega_{e} S_{z, e}-\omega_{n} S_{z, n^{\prime}}+D S_{z, e}^{2}
$$

is treated exactly, and the perturbation is given by

$$
H_{\mathrm{sym}}^{\prime}=A\left(\mathbf{S}_{n} \cdot \mathbf{S}_{e}+\mathbf{S}_{n^{\prime}} \cdot \mathbf{S}_{e}\right) .
$$

As $H_{0, \text { sym }}$ is diagonal in the computational basis (defined as $\left\{\left|e n_{1} n_{2}\right\rangle \mid e=T_{ \pm}, T_{0}\right.$ and $\left.\left.n_{1 / 2}=\uparrow, \downarrow\right\}\right)$ and $\left\langle\downarrow \uparrow\left|H_{0, \text { sym }}\right| \downarrow \uparrow\right\rangle=$ $\left\langle\uparrow \downarrow\left|H_{0, \text { sym }}\right| \uparrow \downarrow\right\rangle$, degenerate perturbation theory is required. However, since $\left\langle T_{j} Y\left|H_{\text {sym }}^{\prime}\right| T_{l} Z\right\rangle=0$ for $j, l=-, 0,+$ and $Y, Z=\downarrow \uparrow, \uparrow \downarrow$, the degeneracy is not removed in the first order. To find the correct zeroth-order wave functions, we need to solve the corresponding secular equations to second order [26]. 
Making use of assumption (3) we then get the second-order eigenenergies,

$$
\begin{gathered}
E_{j, 1}=E_{j, 3}-\varepsilon_{j}, \quad E_{j, 2}=-j \omega_{e}+|j| D, \\
E_{j, 3}=E_{j, 2}-\delta_{j}, \quad E_{j, 4}=E_{j, 2}+\varepsilon_{j},
\end{gathered}
$$

with the corresponding eigenvectors up to first order (see Fig. 3),

$$
\begin{aligned}
& \left|E_{j, 1}\right\rangle=\left|T_{j} \downarrow \downarrow\right\rangle, \quad\left|E_{j, 2}\right\rangle=\frac{1}{\sqrt{2}}\left(-\left|T_{j} \downarrow \uparrow\right\rangle+\left|T_{j} \uparrow \downarrow\right\rangle\right), \\
& \left|E_{j, 4}\right\rangle=\left|T_{j} \uparrow \uparrow\right\rangle, \quad\left|E_{j, 3}\right\rangle=\frac{1}{\sqrt{2}}\left(\left|T_{j} \downarrow \uparrow\right\rangle+\left|T_{j} \uparrow \downarrow\right\rangle\right),
\end{aligned}
$$

and where we have defined the following quantities for compactness:

$$
\begin{gathered}
\varepsilon_{-}=\omega_{n}-A+2 a_{-}, \varepsilon_{0}=\omega_{n}+2 a_{+}, \quad \text { and } \quad \varepsilon_{+}=\omega_{n}+A ; \\
\delta_{-}=-2 a_{-}, \quad \delta_{0}=-2 a_{0}, \quad \text { and } \quad \delta_{+}=2 a_{+} \\
a_{ \pm}=\frac{1}{2} \frac{A^{2}}{\mp D+\omega_{e}+\omega_{n}} \quad \text { and } \quad a_{0}=a_{+}-a_{-}
\end{gathered}
$$

Based on this spectrum, we now approximate $H_{\text {sym }}$ in the following way to obtain an effective Hamiltonian:

$$
H_{\text {sym }} \approx V^{\dagger} \operatorname{diag}\left(E_{-1,1}, \ldots, E_{1,4}\right) V=: H_{\text {sym, eff }},
$$

where $V$ is the matrix of the approximate eigenvectors (see Fig. 3),

$$
\left.V=\left(\left|E_{-, 1}\right\rangle\left|\left(E_{-, 2}\right\rangle \cdots\right| E_{+, 4}\right\rangle\right) .
$$

In the computational basis, $H_{\text {sym, eff }}$ consists of three blocks with the following structure:

$$
\left(\begin{array}{llll}
x & & \\
& x & x & \\
& \times & \times & \\
& & & x
\end{array}\right),
$$

where $\times$ denotes a nonzero entry and each block belongs to one of the three spin states $T_{-}, T_{0}$, or $T_{+}$. Correspondingly, we define the three subspaces $\mathscr{T}_{j}$ spanned by the vectors $\left|T_{j} \downarrow \downarrow\right\rangle\left|T_{j} \downarrow \uparrow\right\rangle,\left|T_{j} \downarrow \uparrow\right\rangle,\left|T_{j} \uparrow \downarrow\right\rangle$, and $\left|T_{j} \uparrow \uparrow\right\rangle$, with $j=$ $-, 0,+$. In this paper, the index $j$ denotes the electronic spin state of the excitation, whereas the letter $i$ always indexes the four nuclear spin states.

In writing Eq. (14) we have neglected all matrix elements between subspace $\mathscr{T}_{i}$ and subspace $\mathscr{T}_{j}(i \neq j)$; the effect of these matrix elements is negligible because the electronic Zeeman splitting $\omega_{e}$ is much larger than the nuclear Zeeman splittings. The dynamics in each of the $\mathscr{T}_{j}$ is therefore closed

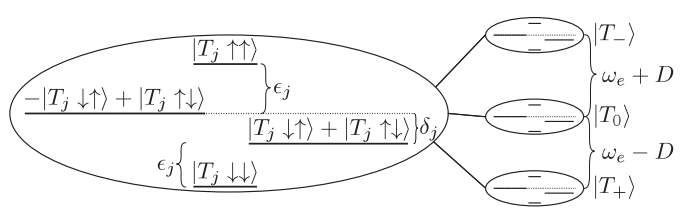

FIG. 3. Eigenspectrum of a symmetric system up to second order of perturbation theory (eigenvectors not normalized). Our basis states obey the relations $S_{z, e}\left|T_{ \pm}\right\rangle=\mp\left|T_{ \pm}\right\rangle, S_{z, e}\left|T_{0}\right\rangle=0, S_{z, n}|\downarrow\rangle=|\downarrow\rangle$, and $S_{z, n}|\uparrow\rangle=-|\uparrow\rangle$. and can be described by an effective $4 \times 4$ Hamiltonian of the form given above. We now analyze the nuclear spin dynamics arising from each of those effective subspace Hamiltonians. A term in the Hamiltonian that is proportional to the identity has no effect on the dynamics within the subspace, so we can subtract $D+\omega_{e}+a_{-}, a_{0}$, and $D-\omega_{e}-a_{+}$from the subspace Hamiltonians for $\mathscr{T}_{-}, \mathscr{T}_{0}$, and $\mathscr{T}_{+}$, respectively (thereby neglecting unimportant phases that are common to all states in each block). We can simplify the resulting Hamiltonians $H_{\text {sym, eff, } j}$ further by transforming to a suitably chosen rotating frame through the transformation

$$
H_{\text {sym, eff }, j}^{\prime}=U_{j} H_{\text {sym, eff, } j} U_{j}^{\dagger}+i U_{j} \frac{d U_{j}^{\dagger}}{d t},
$$

where

$$
U_{j}(t)=R_{z, n}\left(\phi_{j} t\right) \otimes R_{z, n^{\prime}}\left(\phi_{j} t\right)
$$

and

$$
\begin{gathered}
\phi_{ \pm}=-\omega_{n} \mp A-a_{ \pm}, \\
\phi_{0}=-\omega_{n}-a_{-}-a_{+}, \\
R_{z, i}(\phi)=\exp \left(-i S_{z, i} \phi\right) .
\end{gathered}
$$

In this rotating frame we obtain the Hamiltonians

$$
H_{\text {sym, eff, } j}^{\prime}=k(j) 2 a_{j}\left(S_{x, n} S_{x, n^{\prime}}+S_{y, n} S_{y, n^{\prime}}\right),
$$

where $k(j)=1,1,-1$ (with $j=-, 0,+$, as it will be throughout the paper). Each of these effective subspace Hamiltonians therefore induces the dynamics of a direct $X Y$ coupling of the two nuclear spins, with a time evolution corresponding to Rabi flopping between nuclear spin state $|\downarrow \uparrow\rangle$ and nuclear spin state $|\uparrow \downarrow\rangle$. The Rabi frequency $a_{j}$ depends on the spin state $j$ of the optical excitation. According to Eq. (13), we expect $a_{+}$to be fairly similar to $a_{-}$, whereas, in comparison, $a_{0}$ will be much smaller.

We return to the symmetric case to discuss entanglement generation in Sec. IV A but first complete our analysis of the Hamiltonian for nonsymmetric cases.

\section{B. Asymmetric system}

Next we consider an asymmetric system with unequal nuclear Zeeman splittings and/or unequal nuclear-electronic excitation hyperfine coupling. The excited state Hamiltonian is then given by

$$
\begin{aligned}
H_{\text {asym }}=\langle e|H| e\rangle= & -\omega_{n} S_{z, n}+\omega_{e} S_{z, e}-\omega_{n^{\prime}} S_{z, n^{\prime}}+A \mathbf{S}_{n} \cdot \mathbf{S}_{e} \\
& +A^{\prime} \mathbf{S}_{n^{\prime}} \cdot \mathbf{S}_{e}+D S_{z, e}^{2} .
\end{aligned}
$$

For the purpose of applying perturbation theory, we consider the nuclear-spin-mediator coupling as the perturbation and split the Hamiltonian as follows:

$$
\begin{gathered}
H_{0, \text { asym }}=-\omega_{n} S_{z, n}+\omega_{e} S_{z, e}-\omega_{n^{\prime}} S_{z, n^{\prime}}+D S_{z, e}^{2}, \\
H_{\text {asym }}^{\prime}=A \mathbf{S}_{n} \cdot \mathbf{S}_{e}+A^{\prime} \mathbf{S}_{n^{\prime}} \cdot \mathbf{S}_{e} .
\end{gathered}
$$

For the asymmetric system we assume that the parameters $A$ and $A^{\prime}$ and $\omega_{n}$ and $\omega_{n^{\prime}}$ differ sufficiently to fulfill the following two inequalities

$$
\begin{gathered}
\frac{1}{2}\left|A^{\prime}-A \pm\left(\omega_{n^{\prime}}-\omega_{n}\right)\right| \gg\left|a_{ \pm}\right|,\left|a_{ \pm}^{\prime}\right|, \\
\frac{1}{2}\left|\omega_{n^{\prime}}-\omega_{n}\right| \gg\left|a_{0}\right|,\left|a_{0}^{\prime}\right|,
\end{gathered}
$$


where $a_{j}$ and $a_{j}^{\prime}$ are as defined in the previous section, with the latter using $\omega_{n^{\prime}}$ and $A^{\prime}$ rather than $\omega_{n}$ and $A$. Under these assumptions, nondegenerate perturbation theory can be used for calculating the eigensystem. The state corrections to firstorder perturbation theory then introduce a small mixing of computational basis states with corrections of magnitude

$$
\frac{1}{\sqrt{2}} \frac{A}{D \pm\left(\omega_{e}+\omega_{n}\right)} \text { and } \frac{1}{\sqrt{2}} \frac{A^{\prime}}{D \pm\left(\omega_{e}+\omega_{n^{\prime}}\right)},
$$

which are negligible for $A, A^{\prime} \ll \omega_{e}$. The eigenstates of $H_{\text {asym }}$ thus coincide with those of $H_{0 \text {,asym }}$ (which are simply the computational basis states) to a very good approximation.

Analogously to our approach in the symmetric case, we proceed by analyzing individual effective Hamiltonians for the subspaces $\mathscr{T}_{j}(j=-, 0,+)$. After adding a suitable constant to each subspace, we obtain three diagonal Hamiltonians,

$$
H_{\text {asym }, \text { eff }, j}=\phi_{j}^{\prime} S_{z, n^{\prime}}+\phi_{j} S_{z, n},
$$

where $\phi_{j}^{\prime}$ and $\phi_{j}$ are as defined in Eqs. (19) and (20) and the prime denotes that $\omega_{n^{\prime}}$ and $A^{\prime}$ are used for the expression, instead of $\omega_{n}$ and $A$. All three effective Hamiltonians are local, meaning that there is no direct coupling between the two nuclear spins.

\section{Crossover between a symmetric and an asymmetric system}

In a certain region of parameter space neither the symmetric nor the asymmetric analyses are justified. In this section we consider this "crossover" regime, so that in the later discussion we will be able to interpolate between those two cases.

We start with the approximated symmetric Hamiltonian $H_{\text {sym,eff }}$ and treat the asymmetry as the perturbation $H_{\text {co }}^{\prime}$, i.e.,

$$
\begin{gathered}
H_{\mathrm{co}}=H_{\mathrm{sym}, \mathrm{eff}}+H_{\mathrm{co}}^{\prime}, \\
H_{\mathrm{co}}^{\prime}=-\Delta_{1} \omega_{n} S_{z, n^{\prime}}+\Delta_{2} A \mathbf{S}_{n^{\prime}} \cdot \mathbf{S}_{e},
\end{gathered}
$$

where $\Delta_{1}$ is the fractional difference between the two nuclear Zeeman splittings, $\Delta_{1}=\frac{\omega_{n^{\prime}}-\omega_{n}}{\omega_{n}}$, and $\Delta_{2}$ is the fractional difference between the two coupling constants, $\Delta_{2}=\frac{A^{\prime}-A}{A}$.

We begin with the vectors $\left|E_{j, i}\right\rangle$ and values $E_{j, i}{ }^{A}(j=$ $-, 0,+; i=1, \ldots, 4)$ from Sec. III A as the eigenstates and eigenvalues of Hamiltonian $H_{\text {sym,eff }}$. As in the other two cases, we can still neglect those terms of $H_{\text {co }}$ which couple different projections of the excitation spin with strength $\Delta_{2} K / \sqrt{2}$ since the relevant states differ in energy by about $\omega_{e}$, yielding an effective crossover Hamiltonian $H_{\text {co,eff }}$ with the eigenvectors

$$
\begin{gathered}
\left|\widetilde{E}_{j, 1}\right\rangle=\left|T_{j} \downarrow \downarrow\right\rangle, \\
\left|\widetilde{E}_{j, 2}\right\rangle=\frac{1}{\sqrt{2}}\left(\alpha_{j, 2,1}\left|T_{j} \downarrow \uparrow\right\rangle+\alpha_{j, 2,2}\left|T_{j} \uparrow \downarrow\right\rangle\right), \\
\left|\widetilde{E}_{j, 3}\right\rangle=\frac{1}{\sqrt{2}}\left(\alpha_{j, 3,1}\left|T_{j} \downarrow \uparrow\right\rangle+\alpha_{j, 3,2}\left|T_{j} \uparrow \downarrow\right\rangle\right), \\
\left|\widetilde{E}_{j, 4}\right\rangle=\left|T_{j} \uparrow \uparrow\right\rangle,
\end{gathered}
$$

where

$$
\left|\alpha_{j, 2,1 / 2}\right|^{2}=\left|\alpha_{j, 3,2 / 1}\right|^{2}=\frac{1}{2}\left(1 \pm \frac{\operatorname{sgn}\left(a_{j}\right) f_{j}}{\sqrt{a_{j}^{2}+f_{j}^{2}}}\right)
$$

and

$$
f_{ \pm}=\frac{1}{2}\left(\Delta_{2} A \pm \Delta_{1} \omega_{n}\right) \text { and } \quad f_{0}=-\frac{1}{2} \Delta_{1} \omega_{n} .
$$

The corresponding eigenvalues are

$$
\begin{gathered}
\widetilde{E}_{j, 1 / 4}=E_{j, 1 / 4} \pm k(j) f_{j}, \\
\widetilde{E}_{j, 2 / 3}=E_{j, 2}+k(j)\left(a_{j} \mp \operatorname{sgn}\left(a_{j}\right) \sqrt{a_{j}^{2}+f_{j}^{2}}\right),
\end{gathered}
$$

where $k(j)$ is again $1,1,-1$ for $j=-, 0,+$, respectively.

It is easy to see that the eigenstates reduce to those of the symmetric case for a vanishing perturbation $\left(f_{j}=0\right)$, whereas they tend to the computational basis states for a larger perturbation as required for the asymmetric system $\left(\left|f_{j}\right| \gg\left|a_{j}\right|\right)$.

\section{CONTROLLED GENERATION OF ENTANGLEMENT}

The contrasting analyses presented in the previous section suggest that the dynamics of the system will vary significantly depending on its parameters. Therefore, different approaches for achieving controlled entanglement between the two nuclear spins will be required. In this section we show how to do this in each case; we begin with the symmetric system introduced in Sec. III A.

\section{A. Symmetric system: Entangling time evolution}

For the symmetric system, we exploit the effective $X Y$ coupling between spin state $|\uparrow \downarrow\rangle$ and spin state $|\downarrow \uparrow\rangle$ for the generation of entanglement. The fact that the magnitude of this coupling depends on the spin state of the excitation will allow us to control the interaction.

The free time evolution in any of the subspaces $\mathscr{T}_{j}$ takes suitable initial product states of the nuclear spins to entangled states at certain subsequent points of time. In order to quantify the performance of the desired operation, we consider the entangling power of the unitary operator that describes the time evolution of the system. The entangling power is defined as the mean linear entropy produced by the unitary operator acting on a uniform distribution of all (pure) product states [27]. A maximally entangling two-qubit gate, e.g., the controlled-NOT (CNOT) or the controlled-PHASE (CPHASE) gate, possesses an entangling power of $2 / 9$.

For the symmetric system the entangling power of the free time evolution $U_{j}(t)=\exp \left(-i H_{\text {sym,eff, } j}^{\prime} t\right)$ is given by the following simple expression for each of the three subspaces $\mathscr{T}_{j}$ :

$$
e_{j}=\frac{1}{9}\left[3+\cos \left(2 a_{j} t\right)\right] \sin ^{2}\left(a_{j} t\right) .
$$

The entangling power $e_{j}$ is only a function of the coupling strength $a_{j}$ between state $\left|T_{j} \downarrow \uparrow\right\rangle$ and state $\left|T_{j} \uparrow \downarrow\right\rangle$, becoming maximally entangling at times that are odd-integer multiples of $t_{\max }=\pi /\left(2 a_{j}\right)$. However, the characteristic time scale over which entanglement builds up differs significantly between the subspaces, since $a_{ \pm} / a_{0}=\omega_{e} /(2 D)$ to leading order, and this ratio is typically much higher than 1 . Hence, $e_{0}(t) \approx 0$ for $t<\pi /\left(2 a_{ \pm}\right)$when $|D| \ll \omega_{e}$ as we have assumed so far. Figure 4 shows the entangling powers $e_{+}$and $e_{0}$ for a typical ratio of $a_{+} / a_{0}=32$. 


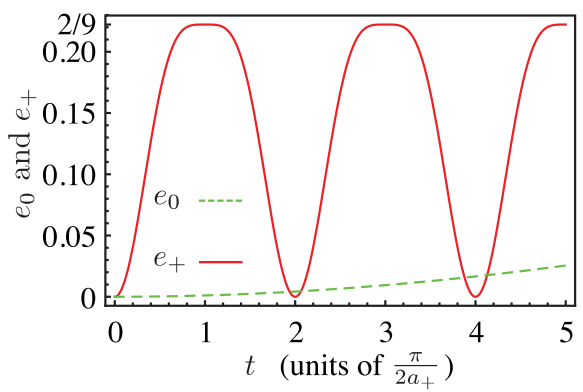

FIG. 4. (Color online) Entangling power $e_{+/ 0}$ of the time evolution operators $U_{+/ 0}(t)$. Here $a_{+}=32 a_{0}$ (see text).

Based on these observations, we can formulate a protocol for the controlled generation of entanglement. Consider a system that is initially in its ground state (i.e., there is no excitation coupling to the nuclear spins). A laser pulse creates the excitation, which will, in general, be in a mixture of the three spin states $\left|T_{-}\right\rangle,\left|T_{0}\right\rangle$, and $\left|T_{+}\right\rangle$. For the present discussion, we assume it to be in state $\left|T_{0}\right\rangle$; the discussion of a mixed initial state follows later. A short microwave pulse allows us to flip the state of the excitation selectively to either the $\left|T_{+}\right\rangle$or the $\left|T_{-}\right\rangle$state since the transition frequencies are split by the zero-field splitting. The entangling dynamics proceeds much more rapidly in these outer subspaces, reaching the first entangling power maximum after a time $t=\frac{\pi}{2 a_{ \pm}}$. We can now apply another microwave pulse to flip the excitation back into the $\left|T_{0}\right\rangle$ state, where the further evolution is much slower and entanglement can be preserved.

There are, however, a few subtle points worth pointing out. First, similar to the case of applying the maximally entangling CNOT operation, only suitable initial states will evolve into entangled states. Second, certain particular input states can reach maximal entanglement in less time than $\frac{\pi}{2 a_{ \pm}}$; for example, the time evolution $U\left(\frac{\pi}{4 a_{+}}\right)$takes $\left|T_{+} \downarrow \uparrow\right\rangle$ to a maximally entangled state.

Finally, the (optical) excitation, whose natural lifetime $\tau$ is assumed to be longer than the time it takes to build up the entanglement $\frac{\pi}{2 a_{ \pm}}<\tau$, needs to be destroyed quickly enough so that no further (slower) evolution unwinding the achieved entanglement occurs in the $\mathscr{T}_{0}$ subspace. This can be achieved either with a coherent optical $\pi$ pulse or, alternatively, by simply waiting for the system to decay back to its ground state if the following hierarchy of time scales exists in the system,

$$
\frac{\pi}{2 a_{ \pm}}<\tau \ll \frac{\pi}{2\left|a_{0}\right|} .
$$

However, in contrast to the de-excitation with a coherent laser pulse, it is not immediately obvious that the nuclear spin entanglement can survive the optical decay process; we therefore now take a small diversion to analyze this decay process in detail.

\section{Decoherence due to the optical decay process}

In general, the optical decay of the mediator induces decoherence on the nuclear spins. In order to quantify this, we make use of the quantum optical master equation (for a full derivation see, e.g., Ref. [28]):

$$
\begin{aligned}
\frac{d}{d t} \tilde{\rho}(t)= & \sum_{\omega, \omega^{\prime}} e^{i\left(\omega^{\prime}-\omega\right) t} \Gamma(\omega)\left[A(\omega) \tilde{\rho}(t) A\left(\omega^{\prime}\right)^{\dagger}\right. \\
& \left.-A\left(\omega^{\prime}\right)^{\dagger} A(\omega) \tilde{\rho}(t)\right]+ \text { H.c., }
\end{aligned}
$$

where $\tilde{\rho}$ denotes the density matrix in the interaction picture, $\Gamma(\omega)$ is the rate for a transition with frequency $\omega$, and H.c. is the Hermitian conjugate. The sum is taken over all optical transitions of the system with transition operators $A(\omega)$ as defined by

$$
A(\omega)=\sum_{E^{\prime}-E=\omega} \Pi(E) \mathscr{D} \Pi\left(E^{\prime}\right),
$$

where $E$ and $E^{\prime}$ are eigenvalues of $H$ that differ by $\omega$ and $\Pi(E)$ denotes the projection onto the eigenspace belonging to eigenvalue $E$. $\mathscr{D}$ denotes the system's optical dipole operator. The symmetric system features 12 optical transitions, $\left|e T_{j} \downarrow \downarrow\right\rangle \rightarrow|0 \downarrow \downarrow\rangle,\left|e T_{j}\right\rangle \frac{1}{\sqrt{2}}(\mp|\downarrow \uparrow\rangle+$ $|\uparrow \downarrow\rangle) \rightarrow|0\rangle \frac{1}{\sqrt{2}}(\mp|\downarrow \uparrow\rangle+|\uparrow \downarrow\rangle)$, and $\left|e T_{j} \downarrow \downarrow\right\rangle \rightarrow|0 \downarrow \downarrow\rangle$, for $j=-, 0,+$. We make the additional assumption that the optical decay rates are all equal and can thus be characterized by a single optical lifetime $\tau$.

Equation (40) can often be simplified by applying an instance of a rotating-wave approximation (RWA), based on the assumption that fast-oscillating terms average out [28], giving this more common form of the quantum optical master equation:

$$
\frac{d}{d t} \tilde{\rho}(t)=\sum_{\omega} \Gamma(\omega)\left[A(\omega) \tilde{\rho}(t) A(\omega)^{\dagger}-A(\omega)^{\dagger} A(\omega) \tilde{\rho}(t)\right]+\text { H.c. }
$$

However, the RWA is only justified when $\left|\omega-\omega^{\prime}\right|^{-1}$ is small compared to the relaxation time of the system $\tau$. Under the assumptions of Eq. (39), this is fulfilled except for the two transition frequencies $\omega_{1}=E_{0,2}+\omega_{0}$ and $\omega_{2}=E_{0,2}+\omega_{0}-\delta_{0}$, which correspond to the transitions $\left|e T_{0}\right\rangle \frac{1}{\sqrt{2}}(\mp|\downarrow \uparrow\rangle+|\uparrow \downarrow\rangle) \rightarrow|0\rangle \frac{1}{\sqrt{2}}(\mp|\downarrow \uparrow\rangle+|\uparrow \downarrow\rangle)$. Therefore we can safely apply the RWA to all remaining frequencies, obtaining

$$
\begin{aligned}
& \frac{d}{d t} \tilde{\rho}(t) \\
& \quad=\sum_{\omega, \omega^{\prime} \in S} e^{i\left(\omega^{\prime}-\omega\right) t} \Gamma(\omega)\left[A(\omega) \tilde{\rho}(t) A\left(\omega^{\prime}\right)^{\dagger}\right. \\
& \left.\quad-A\left(\omega^{\prime}\right)^{\dagger} A(\omega) \tilde{\rho}(t)\right] \\
& +\sum_{\omega \notin S} \Gamma(\omega)\left[A(\omega) \tilde{\rho}(t) A(\omega)^{\dagger}-A(\omega)^{\dagger} A(\omega) \tilde{\rho}(t)\right]+\text { H.c. } \\
& =\sum_{\omega} \Gamma(\omega)\left[A(\omega) \tilde{\rho}(t) A(\omega)^{\dagger}-A(\omega)^{\dagger} A(\omega) \tilde{\rho}(t)\right] \\
& \quad+e^{2 a_{0} i t} \frac{1}{2 \tau}\left[2 A\left(\omega_{1}\right) \tilde{\rho}(t) A\left(\omega_{2}\right)^{\dagger}\right. \\
& \left.\quad-A\left(\omega_{2}\right)^{\dagger} A\left(\omega_{1}\right) \tilde{\rho}(t)-\tilde{\rho}(t) A\left(\omega_{2}\right)^{\dagger} A\left(\omega_{1}\right)\right]+ \text { H.c. }, \quad
\end{aligned}
$$


where $S=\left\{\omega_{1}, \omega_{2}\right\}$ and, using phenomenological decay rates,

$$
\Gamma(\omega)=\left\{\begin{array}{lll}
\frac{1}{2 \tau} & \text { if } & \omega>0, \\
0 & \text { if } & \omega<0 .
\end{array}\right.
$$

This definition of decay rates describes the typical situation of only spontaneous emission occurring, since stimulated emission or absorption of photons is proportional to the negligible power density of thermally activated photons in the environmental modes.

Rather than solving the above master equation for the entire Hilbert space of our system, we are interested here only in the $4 \times 4$ density matrix $\rho^{f}$ of the two nuclear spins after the decay has occurred:

$$
\rho^{f}:=\langle 0|\tilde{\rho}(t=\infty)| 0\rangle .
$$

Limiting the following discussion to this nuclear spin subspace, it is easy to see that only a small number of elements of $\rho^{f}$ can be populated by the decay process. These nonzero elements are $\rho_{a, b}^{f}$ with $a=b=\downarrow \downarrow, a=b=\uparrow \uparrow$, and $a, b \in$ $\{\downarrow \uparrow, \uparrow \downarrow\}$. The final nuclear spin state after the optical decay can be compactly written as

$$
\begin{aligned}
\rho_{a, b}^{f}= & \left\langle e T_{0} a\left|\tilde{\rho}_{0}\right| e T_{0} b\right\rangle+\frac{1}{2} \sum_{j= \pm}\left(\left\langle e T_{j} a\left|\tilde{\rho}_{0}\right| e T_{j} b\right\rangle\right. \\
& \left.+\left\langle e T_{j} \bar{a}\left|\tilde{\rho}_{0}\right| e T_{j} \bar{b}\right\rangle\right),
\end{aligned}
$$

where $\tilde{\rho}_{0}=\tilde{\rho}(0)$ is the full system's density matrix in the interaction picture before the decay process and the bar on top of the nuclear spin states denotes that these are flipped, i.e., $\bar{\downarrow}=\uparrow$ and $\uparrow=\downarrow$. Hence the coherences between $\left|e T_{ \pm} \downarrow \uparrow\right\rangle$ and $\left|e T_{ \pm} \uparrow \downarrow\right\rangle$ do not necessarily survive, but all the coherences between $\left|e T_{0} \downarrow \uparrow\right\rangle$ and $\left|e T_{0} \uparrow \downarrow\right\rangle$ survive the optical decay, reflecting the fact that no RWA approximation has been made for the transitions $\left|e T_{0}\right\rangle( \pm|\downarrow \uparrow\rangle+|\uparrow \downarrow\rangle) \rightarrow|0\rangle( \pm|\downarrow \uparrow\rangle+|\uparrow \downarrow\rangle)$. Physically, this means that these two transitions produce indistinguishable photons due to overlapping emission spectra. In the absence of other decoherence processes, the spectra are simply given by lifetime broadened Lorentzians [29]:

$$
L(\omega)=\frac{1 / \tau}{\left(\omega-\omega_{i}\right)^{2}+(1 / \tau)^{2}} \quad \text { for } \quad i=1,2 .
$$

The photons emitted in all 10 other transitions can, in principle, be distinguished, so that we are effectively dealing with 11 distinct, incoherent decay channels. It is worth noting that there are three decay channels which populate the $|0\rangle( \pm|\downarrow \uparrow\rangle+$ $|\uparrow \downarrow\rangle)$ states, one each for the excitation spin projections $T_{0, \pm}$, but only the decays from the $\mathscr{T}_{0}$ subspace preserve coherence.

In Fig. 5 we plot the purity $\operatorname{Tr}\left[\rho(t)^{2}\right]=\operatorname{Tr}\left[\tilde{\rho}(t)^{2}\right]$ to illustrate the decoherence induced by the optical decay for three different initial density matrices $\rho_{i}(0)=\left|\psi_{i}\right\rangle\left\langle\psi_{i}\right|$ with

$$
\begin{aligned}
& \left|\psi_{1}\right\rangle=\left|e T_{+} \uparrow \downarrow\right\rangle, \quad\left|\psi_{2}\right\rangle=\left|e T_{0} \uparrow \downarrow\right\rangle, \quad \text { and } \\
& \left|\psi_{3}\right\rangle=\frac{1}{2}\left|e T_{0}\right\rangle(|\downarrow \downarrow\rangle+|\downarrow \uparrow\rangle+|\uparrow \downarrow\rangle+|\uparrow \uparrow\rangle) .
\end{aligned}
$$

Solving master equation (43) analytically, we then obtain the following final density matrices of the nuclear spins after the decay:

$$
\rho_{1}^{f}=\frac{1}{2}(|\downarrow \uparrow\rangle\langle\downarrow \uparrow|+| \uparrow \downarrow\rangle\langle\uparrow \downarrow|),
$$

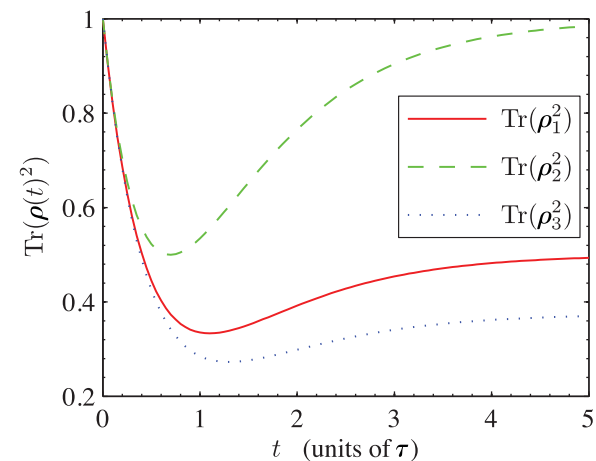

FIG. 5. (Color online) Evolution of the purity $\operatorname{Tr}\left[\rho(t)^{2}\right]$ of a system initialized in an excited state during the optical decay process. The three different initial states shown are defined in (49), and $\tau \delta_{0}=0.05 \ll \pi$.

$$
\begin{aligned}
\rho_{2}^{f}= & \frac{1}{2+2 \delta_{0}^{2} \tau^{2}}\left(\delta_{0}^{2} \tau^{2}|\downarrow \uparrow\rangle\left\langle\downarrow \uparrow\left|+i \delta_{0} \tau\right| \downarrow \uparrow\right\rangle\langle\uparrow \downarrow|\right. \\
& \left.-i \delta_{0} \tau|\uparrow \downarrow\rangle\left\langle\downarrow \uparrow\left|+\left(2+\delta_{0}^{2} \tau^{2}\right)\right| \uparrow \downarrow\right\rangle\langle\downarrow \uparrow|\right), \\
\rho_{3}^{f}= & \frac{1}{4}(\mathbf{1}+|\downarrow \uparrow\rangle\langle\downarrow \uparrow|+| \uparrow \downarrow\rangle\langle\downarrow \uparrow|),
\end{aligned}
$$

with the corresponding purities

$$
\begin{aligned}
& \operatorname{Tr}\left(\rho_{1}^{f}\right)^{2}=1 / 2 \text { and } \operatorname{Tr}\left(\rho_{3}^{f}\right)^{2}=3 / 8, \\
& \operatorname{Tr}\left(\rho_{2}^{f}\right)^{2}=\frac{2+\delta_{0}^{2} \tau^{2}}{2+2 \delta_{0}^{2} \tau^{2}} \stackrel{(39)}{\approx} 1-\frac{\delta_{0}^{2} \tau^{2}}{2} .
\end{aligned}
$$

At first it may seem surprising that an initial state $\left|e T_{+}\right\rangle|\uparrow \downarrow\rangle$ can end up in a complete mixture of $|0\rangle|\downarrow \uparrow\rangle$ and $|0\rangle|\uparrow \downarrow\rangle$. This is due to assumption (39), which underpins the optical master equation (43). This means that we have implicitly included the fast Rabi oscillations between $|\downarrow \uparrow\rangle$ and $|\uparrow \downarrow\rangle$ in the $\mathscr{T}_{+}$ subspace while the system is waiting for the decay. In contrast, in the $\mathscr{T}_{0}$ subspace the inherent dynamics is much slower so that the final result depends on the relative magnitudes of $\delta_{0}$ and $\tau$.

Perhaps surprisingly, decay due to the spontaneous emission of a photon does not act as a source of decoherence if two conditions are met: (i) the system decays from the subspace spanned by the two states $\left|T_{0} \downarrow \uparrow\right\rangle$ and $\left|T_{0} \uparrow \downarrow\right\rangle$; and (ii) $\tau \delta_{0} \ll \pi$, i.e., the energetic splitting of these two states is small compared to the inverse natural lifetime. This property can be turned into a powerful feature for suitable molecular systems, which we exploit in the following.

\section{Dealing with a mixed electronic excitation}

So far, we have assumed that the creation process yields a completely polarized excitation, enabling the simple protocol for the generation of entanglement described in a previous section. Motivated by recent experimental data from a promising candidate molecule [23], we now analyze the implications of having an initial mixture of the states $\left|T_{0}\right\rangle$, and $\left|T_{ \pm}\right\rangle$. Experiments on a ${ }^{13} \mathrm{C}$-labeled methanocarbon of the diethyl malonate monoadduct (DEMF) reveal that the population of the electronic excitation in a sample oriented along the $z$ axis are equally distributed between $\left|T_{+}\right\rangle$and $\left|T_{-}\right\rangle$. In this case the 
lifetime of the excitation also depends on the state of the excitation, being much shorter for the $\left|T_{0}\right\rangle$ state compared to $\left|T_{ \pm}\right\rangle$.

In the following we demonstrate how the generic protocol presented earlier can be adapted to accommodate for the properties of the specific system presented in Ref. [23]. After a short laser pulse for the optical excitation, subspaces $\mathscr{T}_{0, \pm}$ were found to be populated as $p_{-}=0.49, p_{0}=0.02$, and $p_{+}=$ 0.49 with associated lifetimes $\tau_{-}=0.57 \mathrm{~ms}, \tau_{0}=0.02 \mathrm{~ms}$, and $\tau_{+}=0.57 \mathrm{~ms}$. We assume that the nuclear spins are initialized in state $|\downarrow \uparrow\rangle$.

The basic idea of putting the system into $\left|T_{ \pm}\right\rangle$states to let the free time evolution generate entanglement, followed by (mostly) switching off the interaction in the $\mathscr{T}_{0}$ subspace, remains unchanged. As before, switching between different electronic states is accomplished using microwave pulses that are fast on the time scale of the nuclear spin evolution. The adapted protocol proceeds in two stages following the optical excitation. First, we let the desired entanglement build up in the $\mathscr{T}_{+}$subspace by waiting for the time $t=\frac{\pi}{4 a_{+}}$. We then swap the populations of $\left|T_{0}\right\rangle$ and $\left|T_{+}\right\rangle$and wait until the entangled populations have decayed. The difference in decay rates $1 / \tau_{0} \gg 1 / \tau_{ \pm}$means that the population of $\left|T_{-}\right\rangle$largely survives once the majority of $\left|T_{0}\right\rangle$ has decayed to the ground state. Second, population in the $\mathscr{T}_{-}$subspace is maximally entangled at times that are odd-integer multiples of $\frac{\pi}{4 a_{-}}$. We pick the first such point of time after the $\left|T_{0}\right\rangle$ has emptied out and apply another microwave $\pi$ pulse to swap the populations of $\left|T_{0}\right\rangle$ and $\left|T_{-}\right\rangle$. Once more, $\left|T_{0}\right\rangle$ will quickly drain into the ground state, meaning there is now no more excited population left. Ideally, we are left with an almost fully entangled nuclear spin state.

However, the success of the above-described protocol is predicated on the coupling strength $A$ of the nuclear spins to the excitation. In particular, for a very low coupling strength $A$ it takes a long time to entangle the nuclear spins $t \approx \frac{\pi \omega_{e}}{4 A^{2}}$, so that there may be a substantial probability of the optical decay having occurred before the nuclear spins can become properly entangled. In this case the left-hand inequality of Eq. (39) is violated. On the other hand, if the coupling strength $A$ assumes very large values, then the right-hand side of inequality (39) is violated. In the latter case the photons resulting from the transitions $\left|e T_{0}\right\rangle \frac{1}{\sqrt{2}}(\mp|\downarrow \uparrow\rangle+|\uparrow \downarrow\rangle) \rightarrow|0\rangle \frac{1}{\sqrt{2}}(\mp|\downarrow \uparrow\rangle+|\uparrow \downarrow\rangle)$ become distinguishable and the decay is hence no longer coherence preserving. In Fig. 6 we regard the hyperfine coupling $A$ as a tunable parameter and plot the entanglement of formation (EF) [30] of the final state of the two nuclear spins. We consider two different initial states for the mediator spin: a completely polarized state and the mixed state reported in Ref. [23].

\section{Robustness to imperfections in the symmetry of the system}

The previously described protocol for the controlled generation of entanglement assumes a perfectly symmetric system. In the following we analyze the degree of imperfection in the symmetry that may be tolerated. We already know that the crossover between the symmetric and the asymmetric case is not entirely abrupt. In Sect. III C we have found expressions for the eigenstates and eigenvalues that can fully interpolate between the symmetric and the asymmetric case. In this

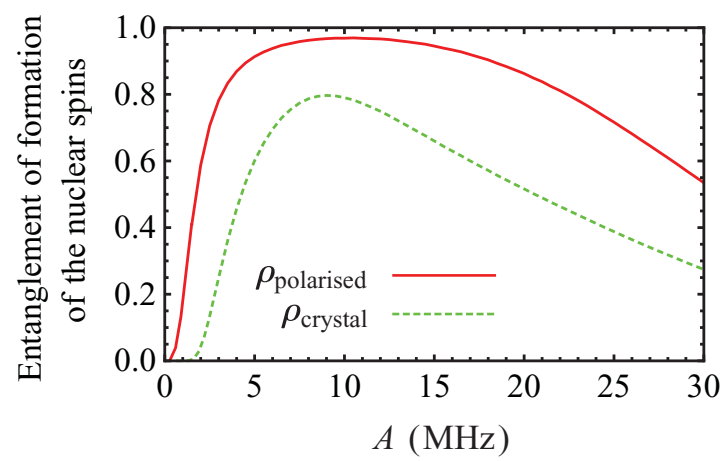

FIG. 6. (Color online) Entanglement of formation of the nuclear spins after applying our protocols as described in the text for different initial polarizations of the excitation: $\rho_{\text {polarized }}=\left|T_{0}\right\rangle\left\langle T_{0}\right|$ (solid line) and $\rho_{\text {crystal }}=0.49\left|T_{-}\right\rangle\left\langle T_{-}|+0.02| T_{0}\right\rangle\left\langle T_{0}|+0.49| T_{+}\right\rangle\left\langle T_{+}\right|$(dashed line). For the first case we used the simple protocol described first in Sec. IV A 2 ; the switching time used is $\frac{\pi}{4 a_{+}}$. In the second case we use the enhanced protocol described at the end of the section; switching times here are $\frac{\pi}{4 a_{+}}$and $\frac{3 \pi}{4 a_{-}}$. The nuclear spins for both curves are assumed to be initially in state $|\downarrow \uparrow\rangle$; for parameters we use the values found in a recent characterization experiment [23]: $D=-296 \mathrm{MHz}$, $\omega_{e}=9.6 \mathrm{GHz}, \omega_{n}=3.7 \mathrm{MHz}, \tau_{-}=0.57 \mathrm{~ms}, \tau_{0}=0.02 \mathrm{~ms}$, and $\tau_{+}=0.57 \mathrm{~ms}$.

crossover case, the effective Hamiltonian still consists of three distinct blocks, each corresponding to the spin state of the excitation $\left|T_{j}\right\rangle(j=-, 0,+)$, and matrix elements connecting the blocks are negligible due to the large energy difference $\omega_{e} \gg\left|\omega_{n}\right|$. This allows us to assign a separate entangling power $\widetilde{e}_{j}$ to the effective Hamiltonians describing each of the blocks.

We now give an easily provable lemma which will enable us to write the relevant analytic expressions of the entangling powers $\widetilde{e}_{j}$ for all three subspaces:

Lemma. Let $H$ be the time-independent Hamiltonian of two spin-1/2 particles with the following two properties.

(1) $\left|E_{1}\right\rangle=|\downarrow \downarrow\rangle, \quad\left|E_{2}\right\rangle=-a|\downarrow \uparrow\rangle+b|\uparrow \downarrow\rangle, \quad\left|E_{3}\right\rangle=$ $b|\downarrow \uparrow\rangle+a|\uparrow \downarrow\rangle$, and $\left|E_{4}\right\rangle=|\uparrow \uparrow\rangle$, with $|a|^{2}+|b|^{2}=1$, and $a, b \in \mathbb{R}$ are eigenvectors of $H$.

(2) The eigenenergies of $H$ satisfy $E_{1}-E_{2}-E_{3}+E_{4}=0$.

Then the entangling power of $U(t)=\exp (-i H t)$ is given by

$e(b, \beta)=\frac{16}{9}\left(b^{2}-b^{4}\right) \sin ^{2}\left(\frac{\beta}{2}\right)-\frac{32}{9}\left(b^{2}-b^{4}\right)^{2} \sin ^{4}\left(\frac{\beta}{2}\right)$,

where $\beta=\left|E_{3}-E_{2}\right| t$. In addition, we have $e(a, \beta)=e(b, \beta)$. Applying the above lemma to our effective Hamiltonians for the crossover case gives three expressions $\widetilde{e}_{j}=e\left(c_{j}, \beta_{j}\right)$, with

$\beta_{j}=2 t \sqrt{a_{j}^{2}+f_{j}^{2}} \quad$ and $\quad c_{j}^{2}=\frac{1}{2}\left(1+\frac{f_{j}}{\sqrt{a_{j}^{2}+f_{j}^{2}}}\right)$.

It is easy to see that the parameter $a_{j}$ now directly competes with the strength of the perturbation $f_{j}$ in the expression for the entangling power. With the measure for the asymmetry

$$
\chi_{j}=\left|\frac{f_{j}}{a_{j}}\right|
$$




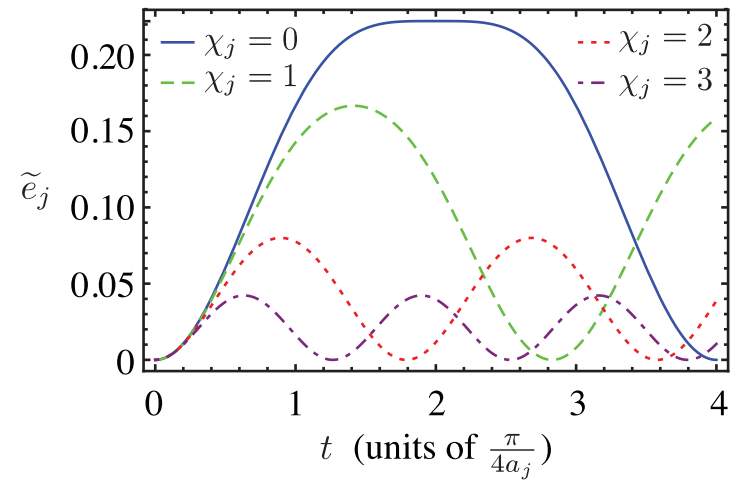

FIG. 7. (Color online) Entangling power $\widetilde{e}_{j}$ of the free time evolution for an asymmetric system, when the excitation is in state $\left|T_{j}\right\rangle$ for different strengths of the asymmetry $\chi_{j}$.

we can write the entangling power compactly as

$$
\tilde{e}_{j}=\frac{\left[3+4 \chi_{j}^{2}+\cos \left(2 a_{j} t \sqrt{1+\chi_{j}^{2}}\right)\right] \sin \left(a_{j} t \sqrt{1+\chi_{j}^{2}}\right)^{2}}{9\left(1+\chi_{j}^{2}\right)^{2}} \text {. }
$$

We plot $\widetilde{e}_{j}$ for different asymmetries $\chi_{j}$ in Fig. 7. Not surprisingly, the entangling power decreases with increasing asymmetry $\chi_{j}$. The maximum of the entangling power, which is achieved for $\beta_{j}=k \pi$, where $k$ is an odd integer, is given by

$$
m_{j}=\frac{16}{9}\left(c_{j}^{2}-c_{j}^{4}\right)-\frac{32}{9}\left(c_{j}^{2}-c_{j}^{4}\right)^{2}=\frac{2}{9} \frac{1+2 \chi_{j}^{2}}{\left(1+\chi_{j}^{2}\right)^{2}} ;
$$

this expression is plotted in Fig. 8. There is no significant reduction in the achievable entanglement power as long as $\chi_{j}<1 / 2$, but the maximum drops quickly outside this regime. We note that for equal hyperfine couplings $\left(\Delta_{2}=0\right)$ but unequal nuclear gyromagnetic ratios,

$$
\frac{\chi_{0}}{\chi_{ \pm}}=\frac{a_{ \pm}}{\left|a_{0}\right|} \approx \frac{\omega_{e}}{2|D|} \gg 1
$$

meaning that the $\mathscr{T}_{0}$ subspace's entangling power $\widetilde{e}_{0}$ is much more affected by the asymmetry than $\widetilde{e}_{ \pm}$. Fortunately, the dynamics in this subspace is also the slowest, so that it can still conveniently serve as a shelf for entangled states generated in $\mathscr{T}_{+}$or $\mathscr{T}_{-}$until the optical excitation has been

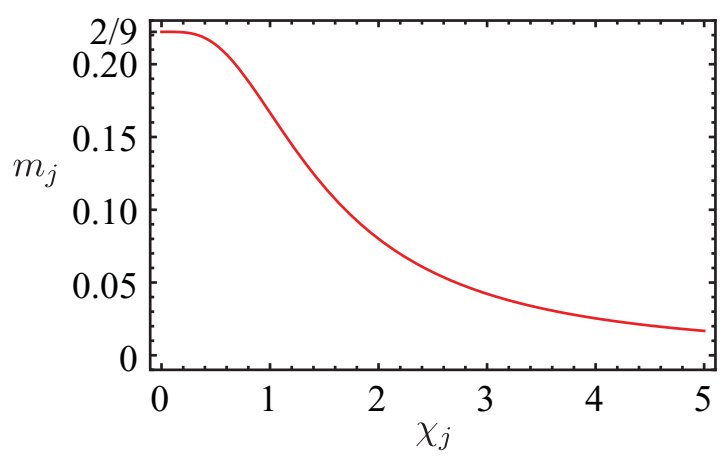

FIG. 8. (Color online) Maximally attainable entangling power $m_{j}$ of the free time evolution, when the excitation is in state $\left|T_{j}\right\rangle$ with respect to the strength of the asymmetry $\chi_{j}$. de-excited or decayed. Importantly, Eq. (59) implies that our scheme is robust against the small deviations from a perfectly symmetrical system which one might expect in realworld experiments. Further, intentionally introduced small differences between frequency $\omega_{n}$ and frequency $\omega_{n^{\prime}}$ (e.g., a chemical shift caused by different surrounding environments) may actually be useful for individual control and tomography of the nuclear spins, while a high-fidelity entangling operation is still possible.

So far we have discussed the behavior of the entangling power in terms of the parameter $\chi_{j}$. While the dependence of $m_{j}$ on $\chi_{j}$ is universal across the three subspaces $\mathscr{T}_{j}$, we obtain qualitatively different results when considering plots that are based directly on the $\Delta_{1 / 2}$ asymmetry parameters. Figure 9 shows the entangling power as a function of $\Delta_{1}$ and $\Delta_{2}$. We see that the behavior is indeed quite different in each of the three subspaces: In the $\mathscr{T}_{-}$subspace we obtain a ridge along which an asymmetry between the nuclear Zeeman splittings and the hyperfine coupling constants completely cancels out, meaning that a perfect operation is possible even for a system that is quite far removed from being symmetric. In contrast, the asymmetries add up in the $\mathscr{T}_{+}$subspace, so that the error tolerance is much reduced in this case. Finally, the $\mathscr{T}_{0}$ subspace is only sensitive to $\Delta_{1}$, i.e., the difference in the nuclear Zeeman splittings without any dependence on the hyperfine constants [see Eq. (35)].

The entangling power vanishes completely in the limit of an entirely asymmetric system [which we take to be defined by inequalities (26) and (27)]. In this case $\chi_{j} \gg 1$, and the eigenstates consequently coincide with the computational basis states, and the eigenvalues are such that the free time evolution no longer generates any entanglement (this result
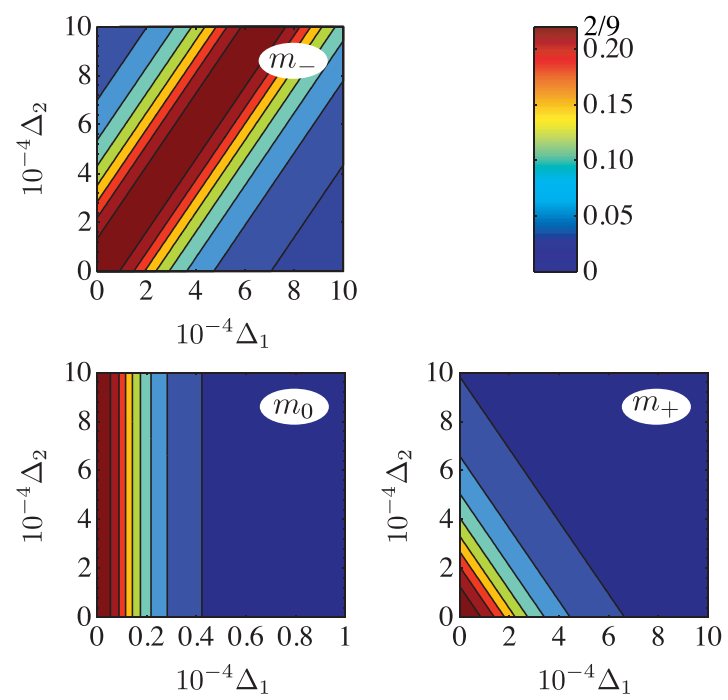

FIG. 9. (Color online) Maximal attainable entangling power $m_{-}$, $m_{0}$, and $m_{+}$as a function of the asymmetry in the Zeeman splitting $\Delta_{1}=\frac{\omega_{n^{\prime}}-\omega_{n}}{\omega_{n}}$ and hyperfine coupling asymmetry $\Delta_{2}=\frac{A^{\prime}-A}{A}$. Other parameters are $A=2.5 \mathrm{MHz}, \omega_{n}=3.7 \mathrm{MHz}, D=-296 \mathrm{MHz}$, and $\omega_{e}=9.6 \mathrm{GHz}$. The entangling power $m_{j}$ only reaches its maximum limit of 2/9 for certain values of $\Delta_{1}$ and $\Delta_{2}$. See the text for a more detailed discussion. 
can be obtained by applying the above lemma). We analyze this situation in the following section.

\section{B. Control methods for the asymmetric system}

For an asymmetric system we cannot rely on the system's free time evolution for the generation of entanglement; this is a direct consequence of the Hamiltonian being decomposable into local Hamiltonians [see Eq. (29)]. Therefore, we need to apply a suitable sequence of radio-frequency and microwave control pulses to accomplish our aim of creating an entangled nuclear spin state. Hence, we proceed by analyzing the dipoleallowed transitions of the asymmetric system (see Fig. 10).

The asymmetric system possesses six (different) nuclear spin transitions on the radio-frequency scale, one per nuclear spin for each of the three spin states of the excitation. Referring to Sec. III B we obtain these from the second-order eigenenergies:

$$
\begin{aligned}
& \omega_{\mathrm{rf}, j}=\left|-j A-\omega_{n}-a_{-}\left(\delta_{j,-}+\delta_{j, 0}\right)-a_{+}\left(\delta_{j, 0}+\delta_{j,+}\right)\right|, \\
& \omega_{\mathrm{rf}, j}^{\prime}=\left|-j A^{\prime}-\omega_{n^{\prime}}-a_{-}^{\prime}\left(\delta_{j,-}+\delta_{j, 0}\right)-a_{+}^{\prime}\left(\delta_{j, 0}+\delta_{j,+}\right)\right|,
\end{aligned}
$$

where $\delta_{k, l}$ is the Kronecker $\delta$, and as before, $j$ indexes the spin state of the excitation $\left|T_{j}\right\rangle$. Further, $\omega_{\mathrm{rf}, j}$ denotes the transition frequency of the first nuclear spin, and $\omega_{\mathrm{rf}, j}^{\prime}$ the transition frequency of the second nuclear spin. In general, all six of these frequencies may be distinct.

Conversely, the spin state of the electronic excitation can be flipped conditional on the nuclear spin state using a suitable microwave pulse. With four nuclear spin states and two excitation spin transitions, this gives a total of eight microwave frequencies taking the excitation from $\left|T_{j}\right\rangle$ to $\left|T_{j^{\prime}}\right\rangle$ with $\left(j, j^{\prime}\right)=(+, 0)$ or $\left(j, j^{\prime}\right)=(0,-)$, or vice versa. These are

$$
\begin{aligned}
& \omega_{\mu \mathrm{w}, \downarrow \downarrow / \uparrow \uparrow}^{j \leftrightarrow j^{\prime}}=\omega_{e}+(-1)^{j} D \pm \frac{1}{2}\left(A+A^{\prime}\right), \\
& \omega_{\mu \mathrm{w}, \downarrow \uparrow / \uparrow \downarrow}^{j \leftrightarrow j^{\prime}}=\omega_{e}+(-1)^{j} D \pm \frac{1}{2}\left(A-A^{\prime}\right) .
\end{aligned}
$$

Here we have neglected second-order perturbation theory shifts proportional to $a_{j}$ and $a_{j}^{\prime}$, as these are typically very small compared to $A$ and $D$.

Several possibilities exist for exploiting this rich transition spectrum in order to realize an entangling operation. In the following we discuss three methods in more detail: a single microwave $2 \pi$ pulse, a pulse sequence of radio- and microwave pulses, and, finally, an adiabatic following method.

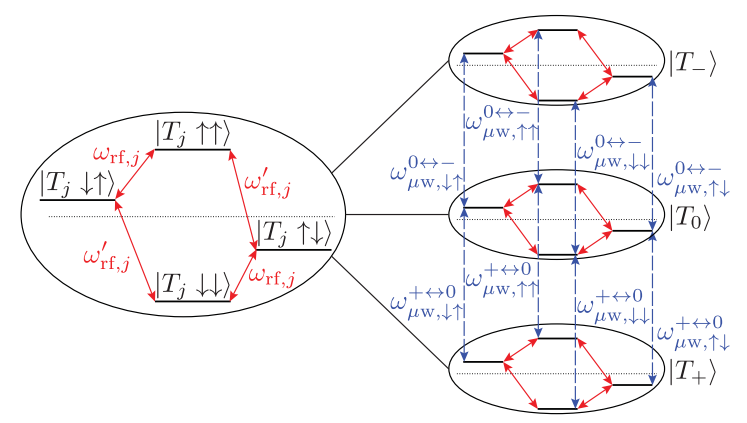

FIG. 10. (Color online) Microwave (dashed line) and radiofrequency transitions (solid line) of the asymmetric system. The computational basis states are eigenstates of the system Hamiltonian [assuming that Eqs. (26) and (27) are fulfilled].

\section{CPHASE gate through a selective $2 \pi$ pulse}

Simply applying a selective $2 \pi$ pulse with the frequency of any of the microwave transitions given in Eqs. (63) and (64) naturally implements a CPHASE gate by imparting a phase of $e^{i \pi}=-1$ to only one of the four nuclear spin states [31].

If the lifetime of the transition were infinite (and in the absence of other spin dephasing mechanisms), the $2 \pi$ pulse could be made perfectly selective, achieved by a pulse that is long in the time domain and, accordingly, spectrally narrow in the frequency domain [32]. However, in practice, the optical lifetime will be finite and this may limit the selectivity and thus the amount of entanglement that can be achieved. The challenge is to find the right balance between a fast pulse that is only partially selective and a slow pulse during which the system suffers from the decoherence induced by the decay. We proceed by analyzing the trade-off that arises from these constraints in the following.

Suppose we are given an initial state that is an equal superposition of the computational basis states and a fully polarized state of the excitation,

$$
\left|\psi_{\text {initial }}\right\rangle=\frac{1}{2}\left|T_{0}\right\rangle(|\downarrow \downarrow\rangle+|\downarrow \uparrow\rangle+|\uparrow \downarrow\rangle+|\uparrow \uparrow\rangle),
$$

and then apply a $2 \pi$ microwave pulse with power $\Omega_{0}$ and with a frequency $\omega_{D}$ corresponding to the energy difference between level $\left|T_{+} \uparrow \uparrow\right\rangle$ and level $\left|T_{0} \uparrow \uparrow\right\rangle$. To describe the dynamics of the excited system we use either the effective asymmetric or the more general effective crossover Hamiltonian, whichever is more appropriate for the precise combination of system parameters in question. In particular, $H_{\text {asym,eff, } j}$ is adequate whenever the eigenvectors closely coincide with the computational basis states, whereas $H_{\mathrm{co}, \mathrm{eff}, j}$ is used otherwise. We apply the following criterion for discriminating between the two cases:

$$
H_{\mathrm{eff}, j}=\left\{\begin{array}{l}
H_{\text {asym,eff, }, j}\left|\alpha_{j, 2,1}\right|^{2} \leqslant 0.001 \text { or }\left|\alpha_{j, 2,1}\right|^{2} \geqslant 0.999, \\
H_{\text {co,eff }, j} \quad 0.001<\left|\alpha_{j, 2,1}\right|^{2}<0.999
\end{array}\right.
$$

with $\alpha_{j, 2,1}$ as defined in Eq. (34). In addition to the effective system Hamiltonian $H_{\text {eff }}$, we need to model the microwave pulse (in the usual RWA), so that the total Hamiltonian during the pulse is given by

$$
\begin{aligned}
H_{\mu \mathrm{w}}= & |0\rangle\left(-\omega_{n} S_{z, n}-\omega_{n^{\prime}} S_{z, n^{\prime}}\right)\langle 0|+| e\rangle H_{\mathrm{eff}}\langle e| \\
& +|e\rangle\left(\Omega_{0} S_{x, e}-\omega_{D} S_{z, e}\right)\langle e| .
\end{aligned}
$$

As this Hamiltonian is time independent, we can use a quantum optical master equation like the one defined in Eq. (43) to model the decay of the excitation in the interaction picture. The transition operators $A(\omega)$ are as defined by Eq. (41), with appropriate projectors onto the eigenspaces of $H_{\mu \mathrm{w}}$. In our calculations we perform the RWA in the master equation (remember that this RWA is different and independent from the RWA for the driving) whenever two frequencies differ by more than $30 \tau^{-1}$, where $\tau$ denotes the lifetime of the excitation.

While optical decay during the application of the pulse may preserve some coherence between the nuclear spin states for specific parameter combinations, this is no longer the case once the pulse has finished: the decay to the ground state for an asymmetric system in the absence of microwave 
driving invariably destroys the nuclear coherences. Therefore, we must use a different approach for taking the system back to its ground state. The first possibility is de-excitation using a resonant optical $\pi$ pulse. Another option is to significantly "speed up" the decay process, e.g., by exciting the system into a different metastable excited state which is known to quickly decay to the ground state. Provided the lifetime of this metastable state is short enough, the wave function of the emitted photon does not carry information about the nuclear spin state, so that the nuclear spin coherence will be preserved. We assume that such a coherence-preserving de-excitation can be accomplished.

As mentioned above, the system is susceptible to potentially harmful decay events during the application of the $2 \pi$ pulse, such that the final nuclear spin state $\rho_{\text {nuc }}$ is a mixture of a population that has spontaneously decayed and the remaining population to which the control sequence has been fully applied. Since we are now dealing with mixed states $\rho_{\text {nuc }}$, the entangling power is no longer a suitable measure for the quality of our operation, and as before we employ the entanglement of formation [30] as an alternative benchmark.

Assuming a simple top-hat pulse profile in the time domain, the pulse duration for a $2 \pi$ pulse is $t=\frac{2 \pi}{\sqrt{2} \Omega_{0}}$, where $\Omega_{0}$ is the applied microwave power. For optimal performance the right balance must be found between pulse selectivity and duration for each combination of system parameters and lifetime $\tau$. We thus maximize the achievable entanglement of formation by varying $\Omega_{0}$ to obtain

$$
E_{F}^{*}=\max _{\Omega_{0}} E_{F}\left(\rho_{\text {nuc }}\right) .
$$

As an example we choose $\tau=10 \mu$ s to plot the quantity $E_{F}^{*}$ in Fig. 11 as a function of $A$ and $A^{\prime}$. Larger hyperfine coupling constants allow a faster selective pulse, and the optimized entanglement of formation of $\rho_{\text {nuc }}$ hence increases with $A$ and $A^{\prime}$. Remarkably, even for a lifetime as short as $10 \mu \mathrm{s}$, a high entanglement of formation can be obtained with only moderate hyperfine coupling strengths.

Finally, we note that the currently presented protocol also works for the symmetric system, where the entanglement operation then only takes a time $t=\frac{\pi}{2 a_{ \pm}} \approx \frac{\pi \omega_{e}}{A^{2}}$, which is much faster than our protocol discussed in Sec. IV A. For a short optical lifetime this approach may thus be advantageous, assuming that a spectrally narrow highly selective $2 \pi$ pulse can be implemented.

\section{Combined microwave and radio-frequency pulse sequence}

In the previous section we discussed a simple implementation of the CPHASE gate that is maximally entangling for suitable system parameters. However, other methods for creating maximally entangled states also exist, and these may be more advantageous if a particular final state is required. Here we briefly discuss an alternative control method that employs a sequence of microwave and radio-frequency pulses instead of a single microwave pulse. Let us assume that we have the initial spin state $\left|T_{0} \downarrow \downarrow\right\rangle$ and would like to create the entangled Bell state $\frac{1}{\sqrt{2}}\left(\left|T_{0} \downarrow \downarrow\right\rangle+\left|T_{0} \uparrow \uparrow\right\rangle\right)$. It is impossible to perform this operation with only radio-frequency pulses. However, we can achieve our aim by "shelving" parts of (a)

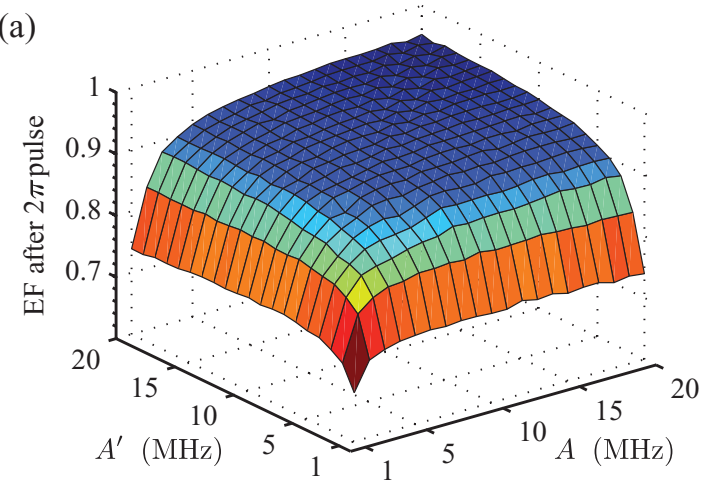

(b)

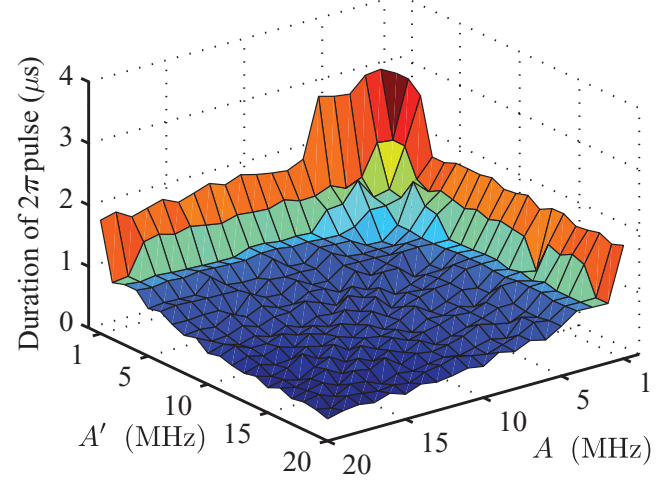

FIG. 11. (Color online) (a) Maximized entanglement of formation of the nuclear spins after the $2 \pi$ pulse with respect to the two hyperfine coupling strengths $A$ and $A^{\prime}$. (b) Optimal duration $t^{*}=\frac{2 \pi}{\sqrt{2} \Omega_{0}^{*}}$ of the microwave $2 \pi$ pulse with respect to the two hyperfine coupling strengths $A$ and $A^{\prime}$. We use the illustrative lifetime $\tau=10_{\mu \mathrm{s}}$, while the other parameters used in this plot are motivated by Ref. [24]: $D=-320 \mathrm{MHz}, \omega_{e}=9.7 \mathrm{GHz}, \omega_{n}=5.97 \mathrm{MHz}$, and $\omega_{n^{\prime}}=14.74 \mathrm{MHz}$.

the population in one of the other electronic spin states. An example of how this approach works in detail is depicted in Fig. 12.

Of course, the microwave and radio-frequency pulses also need to be sufficiently selective for this approach, which imposes a minimal overall pulse sequence duration. Once more, we refrain from discussing sophisticated pulse shaping

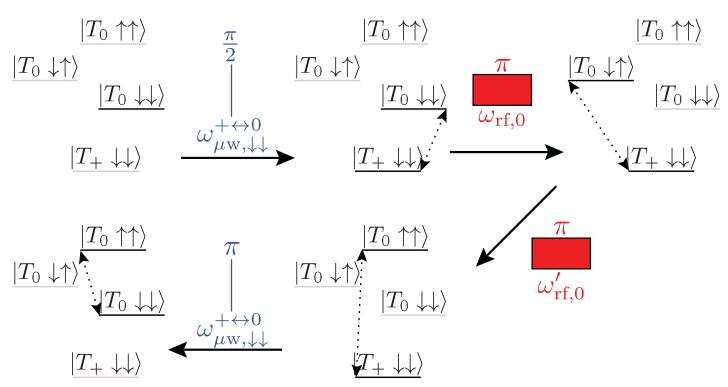

FIG. 12. (Color online) Pulse sequence for the creation of the Bell state $\frac{1}{\sqrt{2}}\left(\left|T_{0} \downarrow \downarrow\right\rangle+\left|T_{0} \uparrow \uparrow\right\rangle\right)$. Black energy levels are populated and gray levels are empty. Dotted arrows denote a coherence between the adjacent energy levels. Only relevant spin levels are shown. 
techniques [32], instead considering Rabi's instructive formula for the transition probability of a driven two-level system,

$$
\mathscr{P}_{1 \rightarrow 2}(t)=\frac{\Omega^{2}}{\Omega^{2}+\Delta^{2}} \sin ^{2}\left(\sqrt{\Omega^{2}+\Delta^{2}} t\right),
$$

where $\Omega$ denotes the strength of the pulse, and $\Delta$ the detuning. We apply this to the closest neighboring transitions of the (resonantly driven) target transition and demand that $\Omega^{2} /\left(\Omega^{2}+\Delta^{2}\right) \ll 1$ for all neighboring transitions, allowing a rough estimation of the duration required for each pulse in the sequence.

We take the example of a recent characterization experiment on an asymmetric system (phosphine oxide fullerene; DMFPH) [24], where the two nuclear spins of interest are provided by a hydrogen and a phosphorus atom in the functional group attached to the fullerene. The hyperfine coupling and the zero-field splitting were measured as

$A \approx 6 \mathrm{MHz}, A^{\prime} \approx 11 \mathrm{MHz}$, and $D \approx-320 \mathrm{MHz}$

at an external magnetic field of $B=0.346 \mathrm{~T}$. Setting an upper bound of 0.01 for the unwanted transition probabilities, the four required pulses can all be applied in less than $1 \mu \mathrm{s}$ for typical nuclear gyromagnetic ratios. The duration of the pulse sequence is thus short compared to the expected optical lifetimes in candidate molecules, so that a high-fidelity entangling operation using this protocol should be feasible. However, we refrain from performing a more detailed analysis as in the previous section, since the results are similar and little additional insight is gained.

\section{Implementation of a CPHASE operation with adiabatic following}

The last method discussed in this paper for creating entanglement in our system relies on the adiabatic following of system eigenstates, similar to the protocol described in Refs. [13] and [33]. Here, it is implemented by slowly modulating the intensity of a microwave pulse that is close to resonance with one or several of the microwave transitions of the excitation spin. Prior to the application of the pulse, the (asymmetric) system is prepared to be in a superposition of computational basis states as follows:

$$
\left|\psi_{\text {initial }}\right\rangle=\left|T_{0}\right\rangle\left(a_{1}|\downarrow \downarrow\rangle+a_{2}|\downarrow \uparrow\rangle+a_{3}|\uparrow \downarrow\rangle+a_{4}|\uparrow \uparrow\rangle\right),
$$

with normalization $\sum_{i=1}^{4}\left|a_{i}\right|^{2}=1$. Starting from this state, the microwave power is then varied such that adiabatic following of instantaneous eigenstates occurs. Once the power is decreased again, all populations return to the computational basis. During the pulse, the eigenstates are energetically shifted and thus pick up a dynamic phase. However, the precise shifts of the states differ due to the the hyperfine coupling, so that, in general, each of the four states acquires a different dynamic phase. This gives rise to an overall combination that can be nontrivial and entangling [33].

Consider applying a microwave field with frequency $\omega_{D}$ whose power envelope is changed gradually following a Gaussian function $\Omega(t)=\Omega_{0} \exp \left[-(t / \sigma)^{2}\right]$. We apply this Gaussian microwave pulse from an initial time $t=-3 \sigma$ to $t=3 \sigma$ and choose the frequency $\omega_{D}$ in such a way that the pulse is off-resonant with all microwave transitions in the system. The diagonal form of the system Hamiltonian $H_{\text {asym,eff }}$ permits a description of the dynamics of each state in the superposition of Eq. (71) with a Hamiltonian connecting all three excitation states of the form

$$
\mathscr{H}_{i}(t)=\langle i|\mathscr{H}| i\rangle=\left(\begin{array}{ccc}
\Delta_{i, 1} & \frac{\Omega(t)}{\sqrt{2}} & 0 \\
\frac{\Omega(t)}{\sqrt{2}} & 0 & \frac{\Omega(t)}{\sqrt{2}} \\
0 & \frac{\Omega(t)}{\sqrt{2}} & \Delta_{i, 2}
\end{array}\right)
$$

written in the basis $\left\{\left|T_{-} i\right\rangle,\left|T_{0} i\right\rangle,\left|T_{+} i\right\rangle\right\}$ for $i=\{\downarrow \downarrow, \downarrow \uparrow$, $\uparrow \downarrow, \uparrow \uparrow\}$ with detunings $\Delta_{i, 1}=\omega_{\mu \mathrm{w}, i}^{0 \leftrightarrow-}-\omega_{D}$ and $\Delta_{i, 2}=$ $\omega_{\mu \mathrm{w}, i}^{+\leftrightarrow 0}-\omega_{D}$. Note that the usual RWA has been performed. Choosing $\omega_{D}$ such that, for each $i$,

$$
\frac{\Omega(t)}{\sqrt{2}} \ll \Delta_{2, i}
$$

then enables us to write an approximate Hamiltonian for each of the nuclear spin states (valid to first order in perturbation theory) as

$$
\mathscr{H}_{i, \text { app }}(t)=\left(\begin{array}{ccc}
\Delta_{i, 1} & \frac{\Omega(t)}{\sqrt{2}} & 0 \\
\frac{\Omega(t)}{\sqrt{2}} & 0 & 0 \\
0 & 0 & \Delta_{i, 2}
\end{array}\right) .
$$

To achieve adiabatic following, the eigenenergies need to be varied slowly to suppress Landau Zener transitions between different eigenstates. Following Ref. [13], this can be accomplished under the following conditions:

$$
\frac{\Omega_{0}}{\Delta_{1, i}^{2}} \ll \sigma \quad \text { for } \quad i=\{\downarrow \downarrow, \downarrow \uparrow, \uparrow \downarrow, \uparrow \uparrow\} .
$$

As we have mentioned above, the net effect achieved by the adiabatic pulse is the acquisition of a phase $\theta_{i}$ for each of the nuclear spin states. The "right" combination of control parameters $\sigma, \Omega_{0}$, and $\Delta_{1, i}$ gives rise to an operation that is locally equivalent to a CPHASE gate if the following condition is met [13]:

$$
\pi=\theta_{1}-\theta_{2}-\theta_{3}+\theta_{4}
$$

In terms of the evolution of nuclear spins, the state $\left|\psi_{\text {initial }}\right\rangle$ has then evolved to

$$
\left|T_{0}\right\rangle\left(a_{1} e^{i \theta_{1}}|\downarrow \downarrow\rangle+a_{2} e^{i \theta_{2}}|\downarrow \uparrow\rangle+a_{3} e^{i \theta_{3}}|\uparrow \downarrow\rangle+a_{4} e^{i \theta_{4}}|\uparrow \uparrow\rangle\right),
$$

corresponding to a local phase on each nuclear spin in addition to the application of a CPHASE gate.

We now address the question of how the optimal combination of control parameters may be found. The dynamical phase that is acquired by a state during the pulse is directly determined by the eigenenergy of the state $\left|T_{0} i\right\rangle$, yielding

$$
\begin{aligned}
\theta_{i} & =-\int_{-3 \sigma}^{3 \sigma} \frac{1}{2}\left(\Delta_{i, 1}-\sqrt{\Delta_{i, 1}^{2}+2 \Omega(t)^{2}}\right) d t \\
& =\frac{\Omega_{0} \sigma}{2} \int_{-3}^{3} \sqrt{\left(\frac{\Delta_{i, 1}}{\Omega_{0}}\right)^{2}+2 \exp \left(-2 x^{2}\right)} d x .
\end{aligned}
$$

Imposing condition (76) and solving for $\sigma=\sigma\left(\omega_{D}, \Omega_{0}\right)$ yields

$$
\sigma=\frac{2 \pi}{\Omega_{0}}\left(\int_{-3}^{3} \sum_{i} \sqrt{\left(\frac{\Delta_{i, 1}}{\Omega_{0}}\right)^{2}+2 \exp \left(-2 x^{2}\right) d x}\right)^{-1},
$$


where the sum is taken over the four nuclear spin eigenstates. To mitigate the effect of decoherence caused by the decay of the excitation, we minimize the duration of the pulse under the constraint that the conditions in Eqs. (73), (75), and (76) are fulfilled, thus obtaining an optimal $\sigma^{*}$.

We incorporate the decoherence caused by the optical decay by modeling the time evolution with the Schrödinger picture master equation

$$
\begin{aligned}
\frac{d}{d t} \rho(t)= & -i\left[\mathscr{H}_{\mathrm{app}}\left(\sigma^{*}\right), \rho(t)\right]+\sum_{\omega} \Gamma(\omega)\left[2 A(\omega) \rho(t) A(\omega)^{\dagger}\right. \\
& \left.-\left\{A(\omega)^{\dagger} A(\omega), \tilde{\rho}(t)\right\}\right]
\end{aligned}
$$

in Lindblad form [28]. $\Gamma(\omega)$ is as defined by Eq. (45) and the Lindblad operators $A(\omega)$ are determined by (41), where the projectors project onto the eigenspaces of $H_{\text {asym,eff }}$ instead of $\mathscr{H}_{\text {app. }}$. This simplification gives 12 (constant) incoherent decay channels, rather than considering time-dependent Lindblad operators and re-evaluating the validity of the RWA at every instance in time (as would be required for the time-dependent Hamiltonian). Effectively, our approach then overestimates the destructiveness of the optical decay, thus giving a lower bound for the entanglement of formation of the nuclear spins.

Figure 13 shows the results of a simulation that applies such an optimized Gaussian pulse with a pulse duration $\sigma^{*}$. For weak coupling strengths $A$ and $A^{\prime}$, this approach achieves a somewhat lower value of the entanglement of formation than the dynamic $2 \pi$ pulse discussed earlier. However, a similarly high-fidelity entangling operation is possible for stronger

(a)

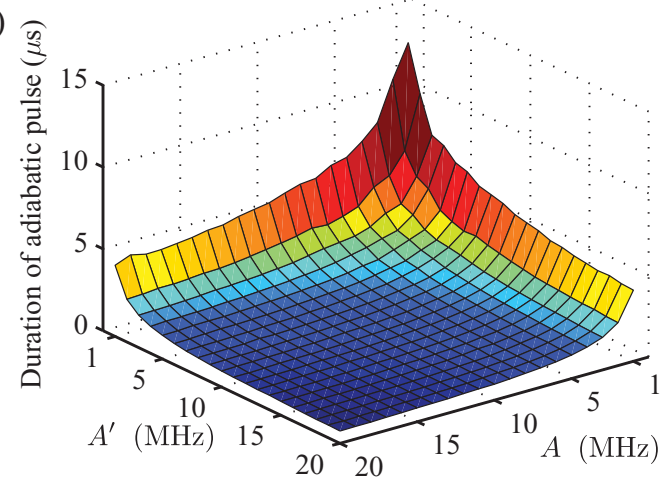

(b)

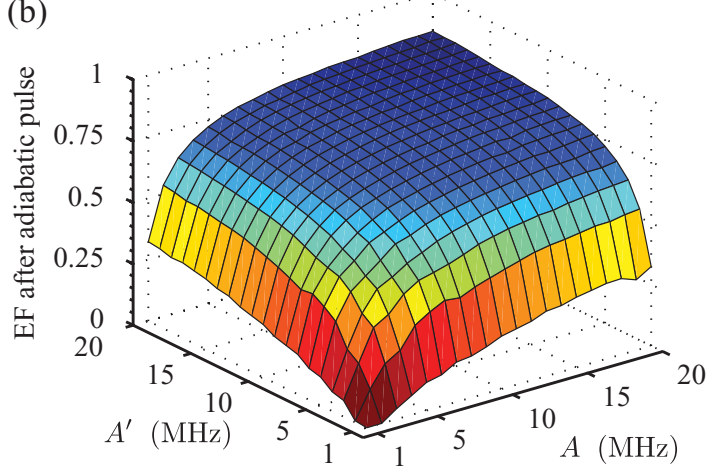

FIG. 13. (Color online) (a) Optimal duration of the adiabatic pulse $6 \sigma^{*}$ optimized over $\omega_{D}$ and $\Omega_{0}$. (b) Entanglement of formation after applying a Gaussian pulse whose duration is characterized by $\sigma^{*}$. As in Fig. 11, other parameters are $\tau=10 \mu \mathrm{s}, D=-320 \mathrm{MHz}$, $\omega_{e}=9.7 \mathrm{GHz}, \omega_{n}=5.97 \mathrm{MHz}$, and $\omega_{n^{\prime}}=14.74 \mathrm{MHz}$. hyperfine coupling. The term "adiabatic following" can invoke the impression that the desired operation will be much slower than a dynamical implementation. It is therefore astounding that our adiabatic pulse takes only about twice as along as the dynamic $2 \pi$ pulse. Finally, we note that the adiabatic method (where the pulse is applied off-resonantly rather than having to hit a specific resonance) is inherently robust against pulse imperfections. This could be a significant advantage for experiments with ensembles of identical molecules. In this case, static and driving field inhomogeneities will inevitably lead to an overor under-rotation of some of the ensemble spins when a dynamical pulse is applied (leaving the wrong excitation subspace populated), whereas the adiabatic approach ensures that all populations end up back in the correct spin levels.

\section{SUMMARY}

In this paper, we have given a detailed analysis showing how a transient optically excited state can be harnessed for the controlled generation of entanglement between two remote nuclear spins. We have identified control methods applicable over a wide range of system parameters and studied their performance with regard to the predominant decoherence mechanism. For a symmetric system consisting of two identical nuclear spins as qubits, the free time evolution is naturally entangling, but the characteristic time scale of the dynamics depends on the state of the excitation and can vary over several orders of magnitude, opening up the possibility of effectively switching the interaction on and off. For an asymmetric system, a different route needs to be taken, and we have presented one adiabatic and two dynamic methods for creating entanglement in this case. We have also included a discussion of the crossover regime between the asymmetric and the symmetric system to establish the robustness of the symmetric operation.

We have shown that the symmetric control method can be remarkably robust against uncertainty or fluctuations in the coupling constants and nuclear Zeeman splittings. As another advantage of the symmetric system, the system can decay back to the ground state without destroying the nuclear spin coherence. Conversely, for the asymmetric system additional control is required for the de-excitation step, yet it is easier to address the nuclear spins individually for single-qubit operations, initialization, and readout.

Interestingly, the active control methods proposed for the asymmetric system are much faster than waiting for the free time evolution in the symmetric case, and they can also be applied to the symmetric system if a short optical lifetime makes this approach advantageous. Finally, we note that the adiabatic control method is intrinsically more robust against control pulse and static field inhomogeneities, making it uniquely suitable for experiments with ensembles of identical systems. Astonishingly, the time required for such an adiabatic operation is only about twice as long as for its dynamical counterpart.

\section{ACKNOWLEDGMENTS}

We thank Vasileia Filidou, Simon C. Benjamin, and John J. L. Morton for fruitful discussions. This work was supported 
by the Marie Curie Early Stage Training network QIPEST (Grant No. MESTCT-2005-020505), the EPSRC through QIP IRC (Grant Nos. GR/S82176/01 and GR/S15808/01), the
National Research Foundation and Ministry of Education, Singapore, the DAAD (German Academic Exchange Service), Linacre College, Oxford, and the Royal Society.
[1] Z.-Y. J. Ou and L. Mandel, Phys. Rev. Lett. 61, 50 (1988).

[2] E. Hagley, X. Maître, G. Nogues, C. Wunderlich, M. Brune, J. M. Raimond, and S. Haroche, Phys. Rev. Lett. 79, 1 (1997).

[3] M. A. Rowe, D. Kielpinski, V. Meyer, C. A. Sackett, W. M. Itano, C. Monroe, and D. J. Wineland, Nature 409, 791 (2001).

[4] T. Wilk, S. C. Webster, A. Kuhn, and G. Rempe, Science 317, 488 (2007).

[5] B. B. Blinov, D. L. Moehring, L.-M. Duan, and C. Monroe, Nature 428, 153 (2004).

[6] M. Stobińska, G. J. Milburn, and L. Stobiński, e-print arXiv:0912.3547 [quant-ph] (2009).

[7] S. C. Benjamin et al., J. Phys. Condens. Matter 18, S867 (2006).

[8] T. D. Ladd, D. Maryenko, Y. Yamamoto, E. Abe, and K. M. Itoh, Phys. Rev. B 71, 014401 (2005).

[9] A. M. Stoneham, A. J. Fisher, and P. T. Greenland, J. Phys. Condens. Matter 15, L447 (2003).

[10] S. C. Benjamin and S. Bose, Phys. Rev. Lett. 90, 247901 (2003).

[11] S. C. Benjamin, B. W. Lovett, and J. H. Reina, Phys. Rev. A 70, 060305 (2004).

[12] S. Ashhab, A. O. Niskanen, K. Harrabi, Y. Nakamura, T. Picot, P. C. de Groot, C. J. P. M. Harmans, J. E. Mooij, and F. Nori, Phys. Rev. B 77, 014510 (2008).

[13] E. M. Gauger, P. P. Rohde, A. M. Stoneham, and B. W. Lovett, New J. Phys. 10, 073027 (2008).

[14] T. Yago, G. Link, G. Kothe, and T.-S. Lin, J. Chem. Phys. 127, 114503 (2007).

[15] D. J. Sloop, H. Yu, T. Lin, and S. I. Weissman, J. Chem. Phys. 75, 3746 (1981).

[16] M. C. J. M. Donckers, A. M. Schwencke, E. J. J. Groenen, and J. Schmidt, J. Chem. Phys. 97, 110 (1992).
[17] V. Zimmermann, M. Schwoerer, and H. C. Wolf, Chem. Phys. Lett. 31, 401 (1975).

[18] G. J. B. van den Berg, D. J. van den Heuvel, O. G. Poluektov, I. Holleman, G. Meijer, and E. J. J. Groenen, J. Magn. Reson. 131, 39 (1998).

[19] J. J. Morton and B. W. Lovett, Annu. Rev. Condens. Matter Phys. 2, 189 (2011)

[20] T. Maly et al., J. Chem. Phys. 128, 052211 (2008).

[21] A. B. Barnes et al., Appl. Magn. Reson. 34, 237 (2008).

[22] J. J. L. Morton, A. M. Tyryshkin, R. M. Brown, S. Shankar, B. W. Lovett, A. Ardavan, T. Schenkel, E. E. Haller, J. W. Ager, and S. A. Lyon, Nature 455, 1085 (2008).

[23] M. Schaffry et al., Phys. Rev. Lett. 104, 200501 (2010).

[24] V. Filidou et al. (in preparation).

[25] P. Neumann, N. Mizuochi, F. Rempp, P. Hemmer, H. Watanabe, S. Yamasaki, V. Jacques, T. Gaebel, F. Jelezko, and J. Wrachtrup, Science 320, 1326 (2008).

[26] L. Schiff, Quantum Mechanics Volume 1 (McGraw-Hill, New York, 1949).

[27] P. Zanardi, C. Zalka, and L. Faoro, Phys. Rev. A 62, 030301(R) (2000).

[28] H. P. Breuer and F. Petruccione, The Theory of Open Quantum Systems (Oxford University Press, New York, 2002).

[29] L. Mandel and E. Wolf, Optical Coherence and Quantum Optics (Cambridge University Press, Cambridge, 1995).

[30] W. K. Wootters, Phys. Rev. Lett. 80, 2245 (1998).

[31] M. A. Nielsen and I. L. Chuang, Quantum Computation and Quantum Information (Cambridge University Press, Cambridge, 2000).

[32] M. A. Bernstein, K. F. King, and X. J. Zhou, Handbook of MRI Pulse Sequences (Elsevier, Amsterdam, 2004).

[33] E. M. Gauger, A. Nazir, S. C. Benjamin, T. M. Stace, and B. W. Lovett, New J. Phys. 10, 073016 (2008). 